\title{
Forwards and pullback behaviour of a non-autonomous Lotka-Volterra system
}

\author{
José A. Langa†, James C. Robinson† Antonio Suárez† \\ $\dagger$ Departamento de Ecuaciones Diferenciales y Análisis Numérico, Universidad de \\ Sevilla, Apdo. de Correos 1160, 41080-Sevilla, Spain. \\ ‡Mathematics Institute, University of Warwick, Coventry, CV4 7AL, U.K. \\ E-mail: langa@numer.us.es; jcr@maths.warwick.ac.uk; suarez@numer.us.es
}

\begin{abstract}
Lotka-Volterra systems have been extensively studied by many authors, both in the autonomous and non-autonomous cases. In previous papers the time asymptotic behaviour as $t \rightarrow \infty$ has been considered. In this paper we also consider the "pullback" asymptotic behaviour which roughly corresponds to observing a system "now" that has already been evolving for a long time. For a competitive system that is asymptotically autonomous both as $t \rightarrow-\infty$ and as $t \rightarrow+\infty$ we show that these two notions of asymptotic behaviour can be very different but are both important for a full understanding of the dynamics. In particular there are parameter ranges for which, although one species dies out as $t \rightarrow \infty$, there is a distinguished time-dependent coexistent state that is attracting in the pullback sense.
\end{abstract}

AMS classification scheme numbers: Primary 37G10, 37G35; Secondary 34D05, 34D23.

Submitted to: Nonlinearity 


\section{Introduction}

In this paper we consider a non-autonomous competitive Lotka-Volterra system for two species $u$ and $v$,

$$
\begin{cases}\dot{u}=u(\lambda-a(t) u-b v) & u(s)=u_{0}>0 \\ \dot{v}=v(\mu-c v-d u) & v(s)=v_{0}>0\end{cases}
$$

where $a(t)$ is continuous,

$$
\lim _{s \rightarrow+\infty} a(s)=0<a(t)<A=\lim _{s \rightarrow-\infty} a(s) \quad \text { for all } \quad t \in \mathbb{R}
$$

and $b, c, d>0$. We will also only consider the case $\lambda, \mu>0$, since when either of these parameters are negative the behaviour is relatively simple. Note that our model equation is asymptotically autonomous $\dagger$ both as $t \rightarrow+\infty$ and as $t \rightarrow-\infty$. We will refer to system (1) as $\mathrm{E}[a(t)]$ : such a notation makes it easier to discuss comparisons of solutions of (1) with the solutions of various autonomous systems $\mathrm{E}[a]$ with $a(t)$ replaced by a constant $a$ (the behaviour of such systems is well understood, e.g. Murray [20], and we recall this as required in what follows).

Since the $u$ and $v$ axes are invariant and solutions of (1) are unique the positive cone $\bar{P}=\{(u, v), u \geq 0, v \geq 0\}$ and its interior $P=\{(u, v), u>0, v>0\}$ are invariant sets. It is therefore consistent to consider only positive solutions, which is also natural in the light of the ecological interpretation of (1) as a model of two competing species.

Various authors have considered non-autonomous versions of the equation concentrating on the asymptotic behaviour as $t \rightarrow+\infty$. However, there is another point of view which, although equivalent in the autonomous case, allows for other arguments in the non-autonomous situation. We study the solutions in terms of the corresponding process $\{S(t, s)\}_{t \geq s}$, where $S(t, s) x_{0}$ represents the solution of the system at time $t$ that was at $x_{0}$ at time $s\left(x_{0} \in \mathbb{R}^{2}\right)$ (cf. Sell [24]). For non-autonomous systems the initial time is as important as the final time: this is in contrast to the autonomous case in which only the time elapsed is relevant, i.e. $S(t, s)=S(t-s, 0)$ for all $t \geq s$. Thus for autonomous systems the behaviour of solutions $S(t, s) x_{0}$ for $t \rightarrow \infty$ is the same as for $s \rightarrow-\infty$. However, this "pullback" behaviour (which forms the basis of the theory of attractors in non-autonomous systems, as developed by Cheban et al. [5], Kloeden and Schmalfuss [12], Schmalfuss [23], Crauel et al. [10]) is distinct from the forwards asymptotics in the non-autonomous case.

In this paper we completely describe the asymptotic behaviour of (1) both as $t \rightarrow \infty$ and as $s \rightarrow-\infty$. The forwards asymptotic behaviour is essentially the same as that of the autonomous system E[0], while the pullback asymptotic behaviour is essentially the same as that of $\mathrm{E}[A]$. For certain parameter ranges these behaviours are distinct,

$\dagger$ We note here that while the theory of asymptotically autonomous equations (developed by Markus [17], Thieme [28], and Mischaikow et al. [18]) gives some useful information about the behaviour of the system as $t \rightarrow \infty$, a full description requires the additional analysis that we present here. 
and the pullback procedure provides more information about the system than would be available solely by considering the forward asymptotics.

The main aim of this paper is not to offer a significant advance to the theory of one particular class of equations (Lotka-Volterra models); rather, it is to illustrate the power of the pullback idea in giving a full description of the dynamics, and to highlight some of the interesting problems that can occur in non-autonomous systems.

\subsection{Previous results for non-autonomous systems}

There are a series of papers that treat non-autonomous $N$-dimensional Lotka-Volterra systems in which all the coefficients are allowed to be non-autonomous (see, among others, Ahmad [2], [3], Ahmad and Lazer [4], Montes de Oca and Zeeman [19], Redheffer [21]). We believe that we could also treat this more general problem, although we have chosen, for the sake of clarity, to consider only the simplest case where the previous methods are not directly applicable.

For (1) the conditions in these papers become

$$
0<a_{1} \leq a(t) \leq a_{2},
$$

for all $t \in \mathbb{R}$ for the results in Ahmad [1] or [2], and

$$
b \mu / c<\lambda<\left[\inf _{t \in \mathbb{R}} a(t)\right] \mu / d
$$

to apply those in Ahmad and Lazer [4] or Montes de Oca and Zeeman [19] (this latter in particular requires that $a(t) \geq a>0$ as in Redheffer [21]). Under these hypotheses the above authors can prove the existence of a strictly positive solution of the system that is globally asymptotically stable, while if

$$
\lambda<b \mu / c \quad \text { or } \quad \lambda>\left[\sup _{t \in \mathbb{R}} a(t)\right] \mu / d
$$

they obtain extinction of one of the species (i.e. the solution goes to zero) as time goes to infinity. In our case none of the conditions (2-4) hold.

\section{Pullback attractors in non-autonomous systems}

We now show how we can consider the solutions of non-autonomous equations within a dynamical systems-like framework, introduce more carefully the pullback idea that we make great use of, and show that the equations in (1) give rise to an order-preserving system.

\subsection{Processes}

We denote by $S(t, s) x$ the solution of (1) at time $t$ that takes the value $x$ at time $s$. Then $S(t, s)$ defines a process, where a general process on a complete metric space $(X, d)$ is a family of mappings $\{S(t, s)\}_{t \geq s}, t, s \in \mathbb{R}$ that satisfy: 
(i) $S(t, t)=\operatorname{Id}_{X}$, for all $t \in \mathbb{R}$,

(ii) $S(t, \tau) S(\tau, s) u=S(t, s) u$ for all $s \leq \tau \leq t, u \in X$, and

(iii) $u \mapsto S(t, s) u$ is continuous in $X$.

\subsection{The pullback attractor}

We now give a formal definition of the "pullback attraction" idea discussed in the introduction. For $A, B \subset X$ we let $\operatorname{dist}(A, B)$ denote the Hausdorff semidistance,

$$
\operatorname{dist}(A, B)=\sup _{a \in A} \inf _{b \in B} d(a, b) \text {. }
$$

Definition 2.1. A time dependent family $\{K(t)\}_{t \in \mathbb{R}}$ is pullback attracting if for each $t \in \mathbb{R}$

$$
\lim _{s \rightarrow-\infty} \operatorname{dist}(S(t, s) D, K(t))=0 .
$$

for every bounded set $D$.

This concept of attraction considers a fixed final time and moves the initial time to $-\infty$ : this does not mean that we are going backwards in time, but rather that we consider the state of the system at time $t$ arising from the same initial condition starting at earlier and earlier times $s$. As remarked above, in the autonomous case this notion of pullback attraction ([12], [23]) is equivalent to the standard definition.

The generalisation of the autonomous concept of invariance is somewhat more straightforward:

Definition 2.2. A family $\{B(t)\}_{t \in \mathbb{R}}$ of subsets of $X$ is said to be invariant with respect to the process $S$ if

$$
S(t, s) B(s)=B(t) \quad \text { for all } \quad(s, t) \in \mathbb{R}^{2}, s \leq t .
$$

The definition of a non-autonomous attractor combines these notions of attraction and invariance.

Definition 2.3. The family of compact sets $\{\mathcal{A}(t)\}_{t \in \mathbb{R}}$ is said to be the global pullback attractor associated to the process $S$ if it is invariant, pullback attracting, and is minimal in the sense that if $\{C(t)\}_{t \in \mathbb{R}}$ is another family of closed pullback attracting sets, then $\mathcal{A}(t) \subset C(t)$ for all $t \in \mathbb{R}$.

(Chepyzhov and Vishik [6] define the concept of kernel sections for non-autonomous dynamical systems: these correspond to the fibres $\mathcal{A}(t)$ in the above definition of a global pullback attractor.)

We now give a general result similar to those that can be found in Crauel et al. [10] and Schmalfuss [23]. The particular form of the theorem given here is modelled on a result for random attractors given in Crauel [9].

Theorem 2.4. There is a global pullback attractor $\{\mathcal{A}(t)\}_{t \in \mathbb{R}}$ if and only if there exists a family $\{K(t)\}_{t \in \mathbb{R}}$ of compact pullback attracting sets. 


\subsection{Complete and hyperbolic trajectories}

The simplest example of an invariant set is a fixed point $x$ with $S(t, s) x=x$ for all $t \geq s$. However, this is a very strong property to require of a solution of a non-autonomous equation. One of the most basic problems with the investigation of non-autonomous systems is to find a sensible generalisation of the notion of a fixed point.

A potential candidate is given by the notion of a complete trajectory, a continuous $\operatorname{map} v: \mathbb{R} \rightarrow X$ with

$$
S(t, s) v(s)=v(t), \quad \text { for all } t \geq s .
$$

This is simply a solution $v(t)$ of the equation that is defined for all $t \in \mathbb{R}$. However, there are of course many such solutions, and we would ideally like to pick out certain "distinguished" solutions. In a previous paper (Langa et al. [14]) we highlighted the importance of complete trajectories with "certain well-defined stability properties" but were less than explicit about what these might be.

One fruitful concept is the notion of a "hyperbolic trajectory" (see Malhotra \& Wiggins [16]) that generalises the idea of a hyperbolic fixed point. We will see in our example that although this concept is useful it is not ideal without some minor modifications. For autonomous systems hyperbolicity can be characterised using the eigenvalues of the linearised equation near the fixed point. In non-autonomous systems

we need to introduce the concept of an exponential dichotomy (see Coppel [8], Sacker \& Sell [22]).

Definition 2.5. Let $A(t)$ be a real $N \times N$ matrix and $\Phi(t, s)$ be the fundamental $N \times N$ matrix solution of

$$
\mathrm{d} X / \mathrm{d} t=A(t) X \quad X(s)=I
$$

so that the solution of $\mathrm{d} \xi / \mathrm{d} t=A(t) \xi$ with $\xi(s)=\xi_{0}$ is given by $\Phi(t, s) \xi_{0}$. Then (5) has an exponential dichotomy if there is a projection operator $P$ and constants $K$ and $\lambda>0$ such that

$$
\begin{aligned}
\|\Phi(t, s) P\| & \leq K \mathrm{e}^{-\lambda(t-s)} & & t \geq s \\
\|\Phi(t, s)(I-P)\| & \leq K \mathrm{e}^{\lambda(t-s)} & & t \leq s .
\end{aligned}
$$

(This definition can be generalised by allowing the projection $P$ to depend on $t$ in such a way that it is invariant, i.e. that

$$
\Phi(t, s) P(s)=P(t) \quad t \geq s
$$

see Siegmund [25] for details.)

Definition 2.6. A complete trajectory $x(t)$ of $\mathrm{d} x / \mathrm{d} t=f(x, t)$ is said to be hyperbolic if the linearised equation

$$
\mathrm{d} X / \mathrm{d} t=\operatorname{D} f(x(t), t) X
$$

has an exponential dichotomy. 
As with the hyperbolicity of fixed points, the hyperbolicity of the trajectory $x(t)$ gives rise to local stable and unstable manifolds which are preserved under perturbation. We reproduce here from [16] only the results that are relevant in this paper: for more details and a proof see $\mathrm{Yi}[29]$. In the statement of the theorem $N_{\delta}(x(t))$ denotes the tubular neighbourhood of $x(t)$ in the space $\mathbb{R}^{N} \times \mathbb{R}$,

$$
N_{\delta}(x(t))=\bigcup_{t \in \mathbb{R}}(B(x(t), \delta), t)
$$

(the notation $B(x, \delta)$ is the open ball in $\mathbb{R}^{N}$ of radius $\delta$ centred at $x$ ).

Theorem 2.7. Let $x(t)$ be a hyperbolic trajectory in $\mathbb{R}^{N}$ such that the projection $P$ from definition 2.5 has rank $k$. Then there exists a $k+1$ dimensional $C^{r}$ manifold $W_{\mathrm{loc}}^{\mathrm{s}}(t)$, an $n-k+1$ dimensional $C^{r}$ manifold $W_{\mathrm{loc}}^{\mathrm{u}}(t)$, and a $\delta$ such that

(i) $W_{\mathrm{loc}}^{\mathrm{s}}$ is positively invariant, while $W_{\mathrm{loc}}^{\mathrm{u}}$ is negatively invariant, and the two manifolds intersect along $x(t)$,

(ii) trajectories on $W_{\mathrm{loc}}^{\mathrm{s}}$ converge towards $x(t)$ exponentially fast as time runs forwards, and leave $N_{\delta}(x(t))$ as time runs backwards, while

(iii) trajectories on $W_{\mathrm{loc}}^{\mathrm{u}}$ leave $N_{\delta}(x(t))$ as time runs forwards but converge exponentially fast towards $x(t)$ as time runs backwards, and

(iv) any trajectory starting in $N_{\delta}(x(t))$ that is not in $W_{\mathrm{loc}}^{\mathrm{s}}$ nor $W_{\mathrm{loc}}^{\mathrm{u}}$ will leave $N_{\delta}(x(t))$ both as time runs forwards and as time runs backwards.

(v) Let $\mathrm{d} x / \mathrm{d} t=f(x, t, p)$ be a family of non-autonomous ODEs that vary with the parameter $p$ in a $C^{r}$ manner (uniformly on sets of the form $K \times \mathbb{R}$ where $K$ is any compact subset of $\mathbb{R}^{N}$ ). Then any hyperbolic trajectory that exists for some value $p=p_{0}$ persists under small perturbations, as do its stable and unstable manifolds: these depend on $p$ in a $C^{r}$ fashion.

We will want to compare the dynamics of our asymptotically autonomous equation with its "limit equation" E[0]. We can treat this case in the context of the above theorem by defining a $C^{1}$ family of functions $\alpha_{p}(t)$ such that

$$
\alpha_{p}(t)=\left\{\begin{array}{cl}
a(1 / p) & t \leq 1 / p \\
a(t) & t \geq(1 / p)+1
\end{array}\right.
$$

and $\alpha_{p}(t)$ is monotonic between $t=1 / p$ and $t=(1 / p)+1$. Then the family of non-autonomous systems $\mathrm{E}\left[\alpha_{p}(t)\right]$ converge towards $\mathrm{E}[0]$ in the appropriate fashion as $p \rightarrow 0$. Since the dynamics of the systems $\mathrm{E}\left[\alpha_{p}(t)\right]$ are the same as those of $\mathrm{E}[a(t)]$ for $t \geq(1 / p)+1$ we expect that perturbations of hyperbolic trajectories of $\mathrm{E}[0]$ will play a role in understanding the dynamics of $\mathrm{E}[a(t)]$.

\subsection{Order preserving systems}

When a non-autonomous system is "order-preserving" (in a sense that we now make precise) it is possible to obtain more information about the structure of its pullback 
attractor. This programme is carried out in some detail in Chueshov [7], Langa \& Suárez [13], and Smith [26].

We say that the process $\{S(t, s): X \rightarrow X\}_{t \geq s}$ is order-preserving if there exists an order relation ' $\preceq$ ' in $X$ such that if $w_{1} \preceq w_{2}$ then $S(t, s) w_{1} \preceq S(t, s) w_{2}$ for all $t \geq s$.

For our equations we can define an appropriate relation $\preceq$ on $\mathbb{R}^{2}$ that makes the system order-preserving: given $\left(u_{1}, v_{1}\right),\left(u_{2}, v_{2}\right)$ we say that

$$
\left(u_{1}, v_{1}\right) \preceq\left(u_{2}, v_{2}\right) \quad \Longleftrightarrow \quad u_{1} \leq u_{2} \quad \text { and } \quad v_{1} \geq v_{2} \text {. }
$$

In fact the following slightly stronger result (cf. Hess and Lazer [11]) will be useful. We will denote the process corresponding to the system $\mathrm{E}[f(t)]$ by $S_{f(t)}(t, s)$, reserving $S(t, s)$ for the process corresponding to $(1)$.

Lemma 2.8. Let $f(t)$ and $\tilde{f}(t)$ be non-negative, and denote by $(u(t), v(t))$ the solution of $\mathrm{E}[f(t)]$ with $(u(s), v(s))=\left(u_{s}, v_{s}\right)$ and by $(\tilde{u}(t), \tilde{v}(t))$ the solution of $\mathrm{E}[\tilde{f}(t)]$ that satisfies $(\tilde{u}(s), \tilde{v}(s))=\left(\tilde{u}_{s}, \tilde{v}_{s}\right)$. Then, provided that $f(t) \geq \tilde{f}(t)$ for all $t \in \mathbb{R}$,

$$
\left(u_{s}, v_{s}\right) \preceq\left(\tilde{u}_{s}, \tilde{v}_{s}\right) \quad \Longrightarrow \quad S_{f(t)}(t, s)\left(u_{s}, v_{s}\right) \preceq S_{\tilde{f}(t)}\left(\tilde{u}_{s}, \tilde{v}_{s}\right)
$$

for all $t \geq s$. We write " $S_{f(t)} \preceq S_{\tilde{f}(t)}$ ".

Proof. Assume initially that $f(t)>\tilde{f}(t)$ for all $t \in \mathbb{R}$. Let $[s, T]$ be the maximal interval on which

$$
(u(t), v(t)) \preceq(\tilde{u}(t), \tilde{v}(t)) \quad \text { for } \quad t \in[s, T]
$$

and suppose that $T<\infty$. At time $T$ one of the following three possibilities occurs:

(i) $u(T)=\tilde{u}(T)=u$ but $v(T)>\tilde{v}(T)$ : clearly for some $\delta_{1}>0$ we have $v(t) \geq \tilde{v}(t)$ for $t \in\left(T, T+\delta_{1}\right]$. We also have

$$
\frac{\mathrm{d}}{\mathrm{d} t}(\tilde{u}-u)(T)=u[(f(T)-\tilde{f}(T)) u+b(v(T)-\tilde{v}(T))] .
$$

Since $v(T)>\tilde{v}(T)$ this is strictly positive and so for some $\delta_{2}>0$ we have $u(t)<\tilde{u}(t)$ for $t \in\left(T, T+\delta_{2}\right]$. This gives $(8)$ on $[s, T+\delta]$, contradicting the maximality of $T$.

(ii) $v(T)=\tilde{v}(T)=v$ but $u(T)<\tilde{u}(T)$ : a similar argument can be used to show that (8) holds on $[s, T+\delta]$ for some $\delta>0$, since we have

$$
\frac{\mathrm{d}}{\mathrm{d} t}(\tilde{v}-v)(T)=v d(u(T)-\tilde{u}(T))<0
$$

(iii) $(u(T), v(T))=(\tilde{u}(T), \tilde{v}(T))=(u, v)$ : when $t=T$ we have (from (9) and (10))

$$
\frac{\mathrm{d}}{\mathrm{d} t}(\tilde{u}-u)(T)=u(f(T)-\tilde{f}(T)) \quad \text { and } \quad \frac{\mathrm{d}}{\mathrm{d} t}(\tilde{v}-v)(T)=0 .
$$

Since $f(T)>\tilde{f}(T)$ we have $\tilde{u}(t)>u(t)$ on $\left(T, T+\delta_{1}\right]$ for some $\delta_{1}>0$. We also have

$$
\frac{\mathrm{d}^{2}}{\mathrm{~d} t^{2}}(\tilde{v}-v)(T)=-v d \frac{\mathrm{d}}{\mathrm{d} t}(\tilde{u}-u)(T)<0,
$$

which implies that $\tilde{v}(t)<v(t)$ on $\left(T, T+\delta_{2}\right]$ for some $\delta_{2}>0$. Once again this gives (8) on the longer interval $[s, T+\delta]$ (with $\delta=\min \left(\delta_{1}, \delta_{2}\right)$ ). 
This proves the result for $f(t)>\tilde{f}(t)$. If $f(t) \geq \tilde{f}(t)$ then for each $\epsilon>0$ apply the above result with $f(t)$ replaced by $f(t)+\epsilon$; noting that the solutions of $\mathrm{E}[f(t)]$ depend continuously on $f(t)$ the result follows as stated by taking the limit as $\epsilon \rightarrow 0$.

\subsection{The separatrix of an autonomous system}

In one of the proofs below we will need to use properties of the separatrix of the autonomous system

$$
\begin{cases}\dot{u}=u(\lambda-a u-b v) & u(0)=u_{0}>0 \\ \dot{v}=v(\mu-c v-d u) & v(0)=v_{0}>0\end{cases}
$$

where $a c<b d$ and $a \mu / d<\lambda<b \mu / c$. In this case there are three fixed points: two stable fixed points at $(0, \mu / c)$ and $(\lambda / a, 0)$, and one saddle point in the interior at

$$
x_{a}^{*}=\frac{1}{b d-a c}(b \mu-c \lambda, d \lambda-a \mu) .
$$

We include a proof since although the system is a standard example in undergraduate differential equations courses, we were unable to find any rigorous treatment in the literature. In the proof we use $(x, y) \geq(u, v)$ to mean $x \geq u$ and $y \geq v$.

Proposition 2.9. If $a c<b d$ and $a \mu / d<\lambda<b \mu / c$ then there is a separatrix $\Gamma_{a}$, given as the graph of a strictly increasing continuous function $\phi_{a}:(0, \infty) \rightarrow(0, \infty)$ with

$$
\lim _{u \rightarrow 0} \phi_{a}(u)=0
$$

such that if

$$
v_{0}\left\{\begin{array}{l}
< \\
= \\
>
\end{array}\right\} \phi_{a}\left(u_{0}\right) \quad \text { then } \quad \lim _{t \rightarrow \infty} S(t, 0)\left(u_{0}, v_{0}\right)=\left\{\begin{array}{c}
(\lambda / a, 0) \\
x_{a}^{*} \\
(0, \mu / c)
\end{array}\right\} .
$$

Furthermore for each fixed $u$ the value of $\phi_{a}(u)$ is continuous in a and is monotonically decreasing in a.

Proof. First we consider the problem for a fixed value of $a$.

Dulac's criterion $\left(\nabla \cdot\left[\frac{1}{u v} \mathbf{f}(u, v)\right]<0\right.$ in $P$, where $\mathbf{f}$ is the right-hand side of $\left.(11)\right)$ shows that the equation has no periodic orbits in $P$, and a simple computation of the time derivative of $\mu|u|^{2}+\lambda|v|^{2}$ shows that all orbits are bounded. It follows from the Poincaré-Bendixson theorem that every orbit converges to one of the fixed points.

Let $\Gamma_{a}$ be the stable manifold of $x_{a}^{*}$, i.e.

$$
\Gamma_{a}=\left\{x: S_{a}(t, 0) x \rightarrow x_{a}^{*} \text { as } t \rightarrow+\infty\right\} .
$$

This is the global extension of the local stable manifold of $x_{a}^{*}$, which is tangent to the linear stable manifold at $x_{a}^{*}$; elementary considerations of the linearisation show that the linear stable manifold moves into $(u, v)>x_{a}^{*}$ and $(u, v)<x_{a}^{*}$. Since $\dot{u}$ and $\dot{v}$ are 
both positive [negative] when $(u, v)>x_{a}^{*}\left[(u, v)<x_{a}^{*}\right]$ it follows that close to $x_{a}^{*}$ the separatrix $\Gamma_{a}$ is the graph of a strictly increasing continuous function $\phi_{a}$; the continuity of $\phi_{a}$ in $a$ within this neighbourhood is a standard result from the theory of local stable manifolds.

The global stable manifold consists of two trajectories. In order to understand their behaviour outside a neighbourhood of $x_{a}^{*}$ we consider the time-reversed flow, writing $\check{u}(t)$ for $u(-t)$. Then since $\dot{\tilde{u}}$ and $\dot{\tilde{v}}$ can be bounded below for $(u, v) \geq x_{a}^{*}+(\epsilon, \epsilon)$, and if $\check{v}(t) \rightarrow \infty$ then we must also have $\check{u}(t) \rightarrow \infty$ (if $\check{u}(t) \rightarrow c<\infty$ then $\dot{\tilde{u}} \rightarrow \infty$, a contradiction) the stable manifold $\Gamma_{a}$ extends to infinity in such a way that $\phi_{a}$ is defined for every $u>\left[x_{a}^{*}\right]_{1}$. Since $(\check{u}, \check{v})<x_{a}^{*}$ is invariant for the time reversed flow and this region contains no periodic orbits (Dulac's criterion again) it follows that the trajectory to the left of $x_{a}^{*}$ converges to the origin as $t \rightarrow-\infty$.

The convergence of $S(t, 0)\left(u_{0}, v_{0}\right)$ to one of the fixed points on the axes when the initial condition lies above/below $\Gamma_{a}$ is now immediate, since $\Gamma_{a}$ is invariant and consists of all points attracted to $x_{a}^{*}$.

The continuity of $\phi_{a}(u)$ for all values of $u$ is a consequence of the continuous dependence of solutions on initial conditions and on the parameter $a$, and it only remains to show that $\phi_{a}(u)$ is monotonically decreasing. This will follow if we can show that for distinct values of $a \neq \tilde{a}$ the separatrices $\Gamma_{a}$ and $\Gamma_{\tilde{a}}$ are disjoint, since $x_{a}^{*} \in \Gamma_{a}$ is strictly increasing with respect to the order $\preceq$ (this follows from a simple calculation of $\left.\mathrm{d} x_{a}^{*} / \mathrm{d} a\right)$. Without loss of generality assume that $a>\tilde{a}$, and suppose that $x \in \Gamma_{a} \cap \Gamma_{\tilde{a}}$. Using lemma 2.8 we have $S_{a} \preceq S_{\tilde{a}}$, and so

$$
x_{a}^{*}=\lim _{t \rightarrow \infty} S_{a}(t, 0) x \preceq \lim _{t \rightarrow \infty} S_{\tilde{a}}(t, 0) x=x_{\tilde{a}}^{*}
$$

However, $x_{a}^{*} \succ x_{\tilde{a}}^{*}$, a contradiction. So $\Gamma_{a} \cap \Gamma_{\tilde{a}}=\emptyset$ as required.

\subsection{A non-autonomous logistic equation}

Also useful will be the following simple result that gives some properties of the solutions of the non-autonomous logistic equation

$$
\mathrm{d} x / \mathrm{d} t=x(p(t)-l(t) x) \quad x(s)=x_{0},
$$

with $p(t)>0$ and $l \in C^{0}(\mathbb{R})$ with $l(t)>0$ for all $t \in \mathbb{R}$. We denote the solution of this equation as $\theta_{[p(\cdot), l(\cdot)]}\left(t, s ; x_{0}\right)$, and note that it can be given explicitly by

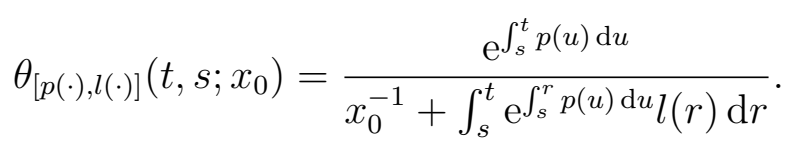

From here it is easy to deduce the following properties:

Lemma 2.10. The solutions of (12) have the following properties:

(i) If $p(t) \rightarrow p>0$ and $l(t) \rightarrow 0$ as $t \rightarrow \infty$ then $\theta_{[p, l]}(t, s) \rightarrow \infty$ as $t \rightarrow \infty$. 
(ii) If $p_{s}(\cdot) \rightarrow p>0$ (uniformly on compact subsets of $\mathbb{R}$ ) as $s \rightarrow-\infty$ then there exists a solution $\alpha: \mathbb{R} \rightarrow \mathbb{R}$ of

$$
\mathrm{d} x / \mathrm{d} t=x(p-l(t) x)
$$

(given explicitly by

$$
\alpha(t)=\frac{\mathrm{e}^{p t}}{\int_{-\infty}^{t} l(\tau) \mathrm{e}^{p \tau} \mathrm{d} \tau},
$$

cf. (13)) such that

$$
\lim _{s \rightarrow-\infty} \theta_{\left[p_{s}(\cdot), l(\cdot)\right]}\left(t_{0}, s\right)=\alpha\left(t_{0}\right) \quad \text { for all } \quad t_{0} \in \mathbb{R}
$$

(Note that in part (ii) $p_{s}(t)$ is a function of $t$ that also depends on $s$. This enables us to consider the pullback behaviour of, for example, $\dot{v}=v((\mu-d u)-c v)$, for fixed initial conditions $\left(u_{0}, v_{0}\right)$ when we know that the $u$ component of $S(t, s)\left(u_{0}, v_{0}\right)$ converges to zero as $s \rightarrow-\infty$.)

\section{Forward asymptotic behaviour}

In this section we analyse the asymptotic behaviour of (1) as $t \rightarrow \infty$. Perhaps unsurprisingly this behaviour is essentially the same as the autonomous system with $a=0$. Indeed, our non-autonomous equation (1) is "asymptotically autonomous" in the sense of Markus [17] with limiting equation E[0]:

$$
\begin{cases}\dot{u}=u(\lambda-b v) & u(s)=u_{0}>0 \\ \dot{v}=v(\mu-c v-d u) & v(s)=v_{0}>0 .\end{cases}
$$

For this autonomous equation, when $\lambda>b \mu / c$ all trajectories converge to $(+\infty, 0)$, while for $0<\lambda<b \mu / c$ there is an interior fixed point $x^{*}=(b \mu-\lambda c, \lambda d) / b d$, whose stable manifold forms a separatrix between solutions tending to $(0, \mu / c)$ and $(+\infty, 0)$. We now recover similar behaviour for the non-autonomous equation.

In this section and the following we denote the process generated by the solutions of (1) by $S(t, s)$.

\subsection{When $\lambda>b \mu / c$}

When $\lambda>b \mu / c$ trajectories are asymptotically unbounded. Since we wish to be specific about the way in which the trajectories diverge we cannot appeal to the results of Markus [17].

Lemma 3.1. If $\lambda>b \mu / c$ then $S(t, s)\left(u_{0}, v_{0}\right) \rightarrow(\infty, 0)$ as $t \rightarrow \infty$.

Proof. We compare trajectories of E $[a]$ with those of two autonomous systems. First we control the long-time behaviour by choosing $k>\max (A, d \lambda / \mu, b d / c)$ and considering the system $\mathrm{E}[k]$. By our choice of $k>d \lambda / \mu$ we know that $\mathrm{E}[k]$ has an attracting fixed 
point $\left(u^{*}, v^{*}\right)$ within $P$, while since $k>A$ we know that $S_{k}(t, s)\left(u_{0}, v_{0}\right) \preceq S(t, s)\left(u_{0}, v_{0}\right)$ for all $t \geq s$. In particular given an $\epsilon>0$ any solution satisfies

$$
\left(u^{*}-\epsilon, v^{*}+\epsilon\right) \preceq S(t, s)\left(u_{0}, v_{0}\right)
$$

for all $t$ sufficiently large.

Now we compare trajectories with those of the system $\mathrm{E}[\delta]$, choosing $\delta$ small enough to ensure that the point $\left(u^{*}-\epsilon, v^{*}+\epsilon\right)$ is contained in the basin of attraction of $(+\infty, 0)$ for $S_{\delta}(\cdot, \cdot)$ : since $S_{\delta}(t, s) \preceq S(t, s)$ for $t, s$ large enough it now follows that $S(t, s)\left(u_{0}, v_{0}\right) \rightarrow(+\infty, 0)$ as $t \rightarrow \infty$.

\subsection{When $0<\lambda<b \mu / c$}

When $0<\lambda<b \mu / c$ we obtain a "non-autonomous separatrix". In what follows we will denote the interior fixed point for the system $\mathrm{E}[0]$ by $x^{*}$ (see above).

Proposition 3.2. For $0<\lambda<b \mu / c$ there exists a time dependent family of continuous functions $\phi_{t}(u): \mathbb{R} \times \mathbb{R}^{+} \rightarrow \mathbb{R}^{+}$which are strictly increasing in $u$ for each fixed $t$ such that if

$$
v\left\{\begin{array}{l}
< \\
= \\
>
\end{array}\right\} \phi_{s}(u) \quad \text { then } \quad S(t, s)(u, v) \rightarrow\left\{\begin{array}{c}
(\infty, 0) \\
x^{*} \\
(0, \mu / c)
\end{array}\right\} \quad \text { as } t \rightarrow \infty .
$$

All limits in the proof are taken as $t \rightarrow \infty$ unless otherwise stated, and we use the notation $\left[\left(x_{1}, x_{2}\right)\right]_{j}=x_{j}$.

Proof. First we show that for a fixed $u_{0}$ if $v_{0}$ is small enough then $S(t, s)\left(u_{0}, v_{0}\right) \rightarrow$ $(\infty, 0)$. Choosing $\epsilon$ such that $\epsilon c<b d$ there exists a $T(\epsilon)$ such that for $t \geq T(\epsilon)$ we have $a(t) \leq \epsilon$. Using the invariance of $u \equiv 0$ and continuity with respect to initial conditions we know that for $v_{0}$ small enough $S(T, s)\left(u_{0}, v_{0}\right)$ is contained in the basin of attraction of $(\lambda / \epsilon, 0)$ for $\mathrm{E}[\epsilon]$. Since $S_{\epsilon}(t, s) \preceq S(t, s)$ for $t, s \geq T$ it follows that for such $v_{0}$ we have $\lim _{t \rightarrow \infty}\left[S(t, s)\left(u_{0}, v_{0}\right)\right]_{2} \rightarrow 0$. Part (i) of lemma 2.10 now shows that $\lim _{t \rightarrow \infty}\left[S(t, s)\left(u_{0}, v_{0}\right)\right]_{1}=\infty$.

Now we show that if $v_{0}$ is large enough then $S(t, s)\left(u_{0}, v_{0}\right) \rightarrow(0, \mu / c)$. Comparing solutions of (1) with those of $\mathrm{E}[0]$ shows that for $v_{0}$ sufficiently large we have $S(t, s)\left(u_{0}, v_{0}\right) \preceq S_{0}(t, s)\left(u_{0}, v_{0}\right)$ where $S_{0}(t, s)\left(u_{0}, v_{0}\right) \rightarrow(0, \mu / c)$. The theory of asymptotically autonomous equations developed by Thieme [28] guarantees in our case (since none of the fixed points of $\mathrm{E}[0]$ are cyclically chained to each other) that the $\omega$ limit set of any bounded solution of $\mathrm{E}[a(t)]$ is one of the fixed points of $\mathrm{E}[0]$. It follows that we must have $S(t, s)\left(u_{0}, v_{0}\right) \rightarrow(0, \mu / c)$.

The two functions

$$
\phi_{s}^{-}(u)=\sup \{v \geq 0: S(t, s)(u, v) \rightarrow(\infty, 0)\}
$$


and

$$
\phi_{s}^{+}(u)=\inf \{v \geq 0: S(t, s)(u, v) \rightarrow(0, \mu / c)\}
$$

are therefore both well-defined and finite $\left(0<\phi^{ \pm}<\infty\right)$ for each $u>0$.

The order-preserving property shows both that $\phi_{s}^{-}$is non-decreasing and that if $v<\phi_{s}^{-}(u)$ then $S(t, s)(u, v) \rightarrow(+\infty, 0):$ if $S(t, s)(u, v) \rightarrow(+\infty, 0)$ and $(u, v) \preceq(\tilde{u}, \tilde{v})$ then we must have $S(t, s)(u, v) \preceq S(t, s)(\tilde{u}, \tilde{v})$ and so $S(t, s)(\tilde{u}, \tilde{v}) \rightarrow(+\infty, 0)$.

Now suppose that for some $s$ the function $\phi_{s}^{-}(u)$ is not strictly increasing in $u$. Then there exists an interval $\left[u_{1}, u_{2}\right]$ such that $\phi_{s}^{-}(u) \equiv v^{*}$ for all $u \in\left[u_{1}, u_{2}\right]$. In particular there is a portion of the graph of $\phi_{s}^{-}$over which $\dot{u}$ changes monotonically while $\dot{v}$ is strictly decreasing (both with respect to $u$ ). Since the graph of $\phi_{\text {. }}^{-}$is invariant, it follows that the function $\phi_{s^{\prime}}^{-}$is decreasing for some $s^{\prime}$ close to $s$, a contradiction.

By applying similar arguments above to the function

$$
\psi_{s}^{+}(v)=\inf \{u \geq 0: S(t, s)(u, v) \rightarrow(\infty, 0)\}
$$

it follows that $\phi_{s}^{-}(u)$ is continuous in $u$, while what is essentially a repetition of the arguments shows that $\phi_{s}^{+}(u)$ enjoys the same properties, with $v>\phi_{s}^{+}(u)$ implying that $S(t, s)(u, v) \rightarrow(0, \mu / c)$.

We showed above that for fixed $s$ the basins of attraction of both $(+\infty, 0)$ and $(0, \mu / c)$ contain an open neighbourhood of the respective limit. Continuity with respect to initial conditions then guarantees that these basins of attraction are open. It follows that if $\phi_{s}^{-}(u) \leq v \leq \phi_{s}^{+}(u)$ then $(u, v)$ must be attracted to $x^{*}$.

Finally observe that the set $\Gamma(s)=\left\{(u, v): \phi_{s}^{-}(u) \leq v \leq \phi_{s}^{+}(u)\right\}$ is invariant and also given by

$$
\Gamma(s)=\left\{(u, v): S(t, s)(u, v) \rightarrow x^{*}\right\} .
$$

Applying the construction discussed at the end of section 2.3 we can see that the hyperbolic fixed point $x^{*}$ perturbs to give a time-dependent hyperbolic trajectory for $\mathrm{E}[a(t)]$ in the region $t \geq T$ for some $T$ sufficiently large. The one-dimensional stable manifold of this hyperbolic trajectory contains all points that converge to $x^{*}$ as $t \rightarrow \infty$. For $s$ sufficiently large this stable manifold coincides with $\Gamma(s)$. It follows, using the invariance of $\Gamma(\cdot)$ and the fact that $S(t, s)$ is $1-1$ for $t \geq s$, that $\Gamma(t)$ is one-dimensional for all $t \in \mathbb{R}$, and hence that $\phi_{s}^{+}(t)=\phi_{s}^{-}(t)$ for all $t \in \mathbb{R}$.

Thus the asymptotic dynamics as $t \rightarrow \infty$ are essentially the same as those of the limiting system $\mathrm{E}[0]$, and in particular we cannot find a strictly positive solution that attracts as $t \rightarrow \infty$. However, we will see in the next section that the equations do in fact possess a distinguished positive solution that attracts "as $s \rightarrow-\infty$ ".

\section{Pullback asymptotic behaviour}

In this section we investigate the pullback dynamics of our system, finding that they are similar to those of the equation $\mathrm{E}[A]$. In particular there exists a range for the values 
of the parameters $(\lambda, \mu)$ for which we can prove the existence of a complete positive trajectory that is pullback attracting.

\subsection{When $A c<b d$ and $A \mu / d<\lambda<b \mu / c$}

First we consider the case $A c<b d$, for which the behaviour is similar to that of proposition 3.2: in the autonomous case $a(t) \equiv A$ the stable manifold $\Gamma_{A}$ of the interior fixed point

$$
x_{A}^{*}=\frac{1}{b d-A c}(b \mu-c \lambda, d \lambda-A \mu)
$$

forms a separatrix between solutions that tend to $(\lambda / A, 0)$ and $(0, \mu / c)$, as shown in proposition 2.9. The remarkable thing about the non-autonomous system is that here the separatrix is precisely that of the system $\mathrm{E}[A]$ and does not depend on time. This is perhaps less surprising than it at first appears, since with the pullback process we send the initial condition "back to $s=-\infty$ ". However, note that we do not have any equivalent of the attraction to the interior fixed point.

Proposition 4.1. Suppose that $A c<b d$ and $A \mu / d<\lambda<b \mu / c$. Then we have

$$
\lim _{s \rightarrow-\infty} S(t, s) x_{0}=\left\{\begin{array}{l}
(0, \mu / c) \\
(\alpha(t), 0)
\end{array}\right\} \quad \text { if } x_{0} \text { lies } \quad\left\{\begin{array}{l}
\text { above } \\
\text { on or below }
\end{array}\right\} \Gamma_{A} .
$$

Proof. If $x_{0}$ lies below $\Gamma_{A}$ then since $S_{A}(t, s) \preceq S(t, s)$ and $S_{A}(t, s) x_{0} \rightarrow(\lambda / A, 0)$ as $s \rightarrow-\infty$ it follows that

$$
\lim _{s \rightarrow-\infty}\left[S(t, s) x_{0}\right]_{2}=0
$$

and part (iii) of lemma 2.10 implies that

$$
\lim _{s \rightarrow-\infty}\left[S(t, s) x_{0}\right]_{1}=\alpha(t) .
$$

If $x_{0}$ lies above $\Gamma_{A}$ then there exists an $\epsilon$ such that $x_{0}$ lies above $\Gamma_{A-\epsilon}$, since the separatrices $\Gamma_{a}$ move down as $a$ increases (see proposition 2.9). But there exists a $T(\epsilon)$ such that $A-\epsilon \leq a(t)$ for all $t \leq T$, and hence for which

$$
S(t, s) \preceq S_{A-\epsilon}(t-s) \quad \text { for all } \quad t, s \leq T .
$$

Since $x_{0}$ lies above $\Gamma_{A-\epsilon}$ it follows that

$$
\lim _{s \rightarrow-\infty}\left[S(t, s) x_{0}\right]_{1}=0
$$

which in turn (using part (ii) of lemma 2.10) implies that

$$
\lim _{s \rightarrow-\infty} S(t, s) x_{0}=(0, \mu / c)
$$

Now observe that since $(0, \mu / c)$ is a fixed point of $S(t, s)$ and $S(t, s)$ is continuous we must have, for any $t^{\prime}>t$ (and in particular for $t^{\prime}>T(\epsilon)$ )

$$
\lim _{s \rightarrow-\infty} S\left(t^{\prime}, s\right) x_{0}=S\left(t^{\prime}, t\right)\left[\lim _{s \rightarrow-\infty} S(t, s) x_{0}\right]=S\left(t^{\prime}, t\right)(0, \mu / c)=(0, \mu / c) .
$$


Finally we consider the case $x_{0} \in \Gamma_{A}$. Note that since $\Gamma_{A}$ is invariant for $S_{A}$, and $S \succeq S_{A}$, it follows that the portion of $P$ lying on or beneath $\Gamma_{A}$ (and including the origin) is invariant; we now excise a small portion of this region to the left of a vertical line in order to exclude the origin, and denote this region (which is still invariant for $S$ by $P_{\Gamma}$.

We show that the process $S$ restricted to $P_{\Gamma}$ has a pullback attractor. Indeed, since $S(t, s) \succeq S_{A}(t, s)$ we must have

$$
\lim _{s \rightarrow-\infty} S(t, s) x_{0} \succeq x_{A}^{*}
$$

while for each $\epsilon>0$ we can choose a $T(\epsilon)$ such that $A-\epsilon \leq a(t)$ for all $t \leq T$. It follows that for $s \leq t \leq T$ we have $S(t, s) \preceq S_{A-\epsilon}(t, s)$; any $x_{0}$ lies below $\Gamma_{A-\epsilon}$, and so

$$
S_{A-\epsilon}(t, s) \rightarrow\left(\frac{\lambda}{A-\epsilon}, 0\right)
$$

It follows that

$$
\lim _{s \rightarrow-\infty} S(t, s) x_{0} \preceq\left(\frac{\lambda}{A-\epsilon}, 0\right) \quad \text { for all } \quad t \leq T(\epsilon) .
$$

Thus there is a pullback attractor $\mathcal{A}_{\Gamma}(t)$ that lies in the region

$$
x_{A}^{*} \preceq x \preceq\left(\frac{\lambda}{A-\epsilon}, 0\right)
$$

when $t \leq T(\epsilon)$.

Now take a point $x \in \mathcal{A}_{\Gamma}(t)$, and suppose that $x_{2} \neq 0$. Note that it follows that as $s \rightarrow-\infty$

$$
\operatorname{dist}\left(S_{A-\epsilon}(s, t) x, \Gamma_{A-\epsilon}\right) \rightarrow 0
$$

Since $S \preceq S_{A-\epsilon}$ and $\mathcal{A}_{\Gamma}(t)$ is invariant and lies wholly within $P_{\Gamma}$ (so that in particular backwards trajectories are bounded away from the origin), this implies that $S(s, t) x$ lies above $\Gamma_{A}$ for $s$ sufficiently small; but this is impossible. It follows that $x_{2}=0$, and hence, since $S(t, s) x$ is a bounded trajectory of $S$, that

$$
\lim _{s \rightarrow-\infty} S(t, s) x_{0}=(\alpha(t), 0)
$$

We omit the other two cases that occur when $A c<b d$ (that is $\lambda<A \mu / d$ and $\lambda>b \mu / c$ ) since similar behaviour occurs when $A c>b d$ (for $\lambda<b \mu / c$ and $\lambda>A \mu / d$ respectively). Since it is only when $A c>b d$ that we can obtain a coexistent pullback attracting state we treat this case in full, starting with these two parameter ranges. 


\subsection{When $A c>b d$}

Note that the pullback asymptotic dynamics on the $u$-axis (that is, $v \equiv 0$ ) is described by the pullback attractor of the logistic equation $\dot{u}=u(\lambda-a(t) u)$, while for the dynamics on the $v$-axis $(u \equiv 0)$ it is described by the autonomous equation $\dot{v}=v(\mu-c v)$. In the following two lemmas we show that for suitable parameter ranges the attractors on the $u$ or $v$ axis is globally asymptotically stable.

4.2.1. Only one species remains. We start with the case $\lambda<b \mu / c$. Here we have "pullback extinction" for the first species. (The autonomous equation $\mathrm{E}[A]$ has $(0, \mu / c)$ as an attracting fixed point.)

Lemma 4.2. Suppose that $A c>b d$ and $\lambda<b \mu / c$. Then, for all $\left(u_{0}, v_{0}\right) \in P$ we have

$$
\lim _{s \rightarrow-\infty} S(t, s)\left(u_{0}, v_{0}\right)=(0, \mu / c) .
$$

Proof. Under these conditions on the parameters, there exists a $T \in \mathbb{R}$ such that for all $t_{0} \leq T$ we have $a\left(t_{0}\right) c>b d$ and so

$$
\frac{d}{a\left(t_{0}\right)} \lambda<\frac{c}{b} \lambda<\mu \text {. }
$$

All solutions of the system $\mathrm{E}\left[a\left(t_{0}\right)\right]$ satisfy

$$
\lim _{s \rightarrow-\infty} S_{a\left(t_{0}\right)}\left(u_{0}, v_{0}\right)=(0, \mu / c),
$$

while we know from lemma 2.8 that $S(t, s)(u, v) \preceq S_{a\left(t_{0}\right)}(t, s)(u, v)$. It follows that

$$
\lim _{s \rightarrow-\infty}\left[S(t, s)\left(u_{0}, v_{0}\right)\right]_{1}=0
$$

which implies by part (ii) of lemma 2.10 that $\lim _{s \rightarrow-\infty}\left[S\left(t_{0}, s\right)\left(u_{0}, v_{0}\right)\right]=\mu / c$. Since $(0, \mu / c)$ is a fixed point of $S(\cdot, \cdot)$ the limiting behaviour in (17) follows for any $t \in \mathbb{R}$.

We now deal with the extinction of the second species (the autonomous equation $\mathrm{E}[A]$ has $(\lambda / A, 0)$ as an attracting fixed point.)

Lemma 4.3. Suppose that $A c>b d$ and $\lambda>A \mu / d$. Then for all $\left(u_{0}, v_{0}\right) \in P$ we have

$$
\lim _{s \rightarrow-\infty} S(t, s)\left(u_{0}, v_{0}\right)=(\alpha(t), 0)
$$

where $\alpha(t)$ is given in (14) with $p=\lambda$ and $l(t)=a(t)$.

Proof. Comparing solutions of $\mathrm{E}[a(t)]$ with those of $\mathrm{E}[A]$, for which

$$
\lim _{s \rightarrow-\infty} S_{A}(t, s)\left(u_{0}, v_{0}\right)=(\lambda / A, 0)
$$

it follows that $\lim _{s \rightarrow-\infty}\left[S(t, s)\left(u_{0}, v_{0}\right)\right]_{2}=0$. Part (ii) of lemma 2.10 now shows that

$$
\lim _{s \rightarrow-\infty}\left[S(t, s)\left(u_{0}, v_{0}\right)\right]_{1}=\alpha(t)
$$

as claimed. 
4.2.2. A pullback attracting coexistent state. Finally we investigate the parameter range in which we obtain our pullback attracting coexistent state: the autonomous system $\mathrm{E}[A]$ has an attracting interior fixed point for this set of parameters.

In two proofs of this section we will make use of the following result from Ahmad and Lazer [4] (Lemma 3), rewritten here using our order notation.

Lemma 4.4. Suppose that there exist $\delta, \delta_{1}, \delta_{2}>0$ such that for some fixed $t \in \mathbb{R}$

$$
\begin{aligned}
& \delta_{1} a(t)>\delta_{2} d+\delta \\
& \delta_{2} c>\delta_{1} b+\delta,
\end{aligned}
$$

and that there exist solutions $x_{1}(\cdot)$ and $x_{2}(\cdot)$ of $(1)$ such that

$$
a^{-} \preceq x_{i}(s) \preceq a^{+} \quad \text { for all } \quad s \leq t
$$

where $a^{-} \preceq a^{+}$and both are elements of $P$. Then $x_{1}(t)=x_{2}(t)$ for all $t \in \mathbb{R}$.

Theorem 4.5. If $A c>b d$ and

$$
b \mu / c<\lambda<A \mu / d
$$

then there exists a complete trajectory $(U(t), V(t)) \in P$ such that for each $u_{0}, v_{0}>0$ and every $t \in \mathbb{R}$,

$$
\lim _{s \rightarrow-\infty} S(t, s)\left(u_{0}, v_{0}\right)=(U(t), V(t)) .
$$

Proof. For any $\epsilon>0$ such that $(A-\epsilon) c>b d$ there exists a $t_{0}(\epsilon)$ such that $a(t)>(A-\epsilon)$ for all $t \leq t_{0}$. It follows that for $s, t \leq t_{0}$ we have

$$
S_{A}(t, s) \preceq S(t, s) \preceq S_{A-\epsilon}(t, s) .
$$

Every system $\mathrm{E}[a]$ with $A-\epsilon \leq a \leq A$ has an attracting interior fixed point $x_{a}^{*}$, and for the parameter range considered here $x_{a}^{*}$ is decreasing (with respect to the order $\preceq$ ) in $a$.

It follows that

$$
x_{A}^{*} \preceq \lim _{s \rightarrow-\infty} S(t, s)\left(u_{0}, v_{0}\right) \preceq x_{A-\epsilon}^{*} .
$$

Since $S\left(t^{\prime}, t\right) x$ depends continuously on $x$, it follows that for any $t^{\prime}$

$$
S\left(t^{\prime}, t\right) x_{A}^{*} \preceq \lim _{s \rightarrow-\infty} S\left(t^{\prime}, s\right)\left(u_{0}, v_{0}\right) \preceq S\left(t^{\prime}, t\right) x_{A-\epsilon}^{*}
$$

and theorem 2.4 ensures the existence of a non-autonomous attractor $\mathcal{A}(t)$.

Any two trajectories $x_{1}(t)$ and $x_{2}(t)$ in $\mathcal{A}(t)$ must satisfy

$$
x_{A}^{*} \preceq x_{i}(t) \preceq x_{A-\epsilon}^{*}
$$

for $t \leq t_{0}$. Lemma 4.4 now guarantees that $x_{1}(t)=x_{2}(t)$ for all $t \in \mathbb{R}$, and thus $\mathcal{A}(t)$ consists of a single trajectory $(U(t), V(t))$ as claimed. 


\section{Conclusions}

We have described in some detail the dynamics of a two-dimensional non-autonomous competitive Lotka-Volterra model that is asymptotically autonomous both as $t \rightarrow \infty$ and as $t \rightarrow-\infty$. While the asymptotic behaviour as $t \rightarrow \infty$ corresponds to that of the limiting system far in the future, the pullback asymptotic behaviour as $s \rightarrow-\infty$ appears to correspond to that of the limiting system in the distant past. We think that at the very least this example should serve to clarify the type of information that can be picked up using the pullback idea.

If it really is forward asymptotic behaviour that is of interest then we can expect to gain little from the pullback approach, but if a propensity to favour this point of view is a product only of its familiarity (and the equivalence of the two notions in the autonomous case) then the pullback procedure provides another technique that can be useful in uncovering important qualitative features of the dynamics.

For example, for a certain range of parameters there is a distinguished positive "coexistent" trajectory $x(t)=(u(t), v(t))$ that is pullback attracting. To give this a biological interpretation, if were to arrive today $(t=0)$ at a remote island on which two species have been competing according to (1) for a long time, the distribution of the two species would be very close to $(u(t), v(t))$. However, we know that one of the species is destined to die out in the future.

Some interesting mathematical questions are also raised. When $A$ is sufficiently small the system $\mathrm{E}[a(t)]$ will be $C^{1}$ close to $\mathrm{E}[0]$ over the whole line $(t \in \mathbb{R})$ and the saddle point $x^{*}$ of the system $\mathrm{E}[0]$ will become a hyperbolic trajectory for $\mathrm{E}[a(t)]$ : our results confirm this, so that when $A c<b d$ the pullback behaviour and the forwards asymptotic behaviour are similar. However, when $A c>b d$ the picture is different: somehow we have to "join" the pullback behaviour (an attracting coexistent trajectory) to the forwards behaviour: it is not clear that the hyperbolic trajectory emanating from the stable positive fixed point of $\mathrm{E}[A]$ remains hyperbolic for all $t \in \mathbb{R}$. In particular it seems more natural to allow for "eventually hyperbolic" trajectories where we only require an exponentially dichotomy for $t, s \geq T$ or $t, s \leq T$ (for some appropriate $T$ ).

With further analysis we believe that it would have been possible to treat not only the predator-prey and cooperative cases, but also higher-dimensional systems and more general non-autonomous terms (indeed, a related infinite-dimensional problem is studied in Langa et al. [15]). However, we have preferred to keep the problem relatively simple in order to show that the pullback procedure can be a very useful tool.

\section{Acknowledgments}

This work has been partially supported by Proyecto D.G.I.C.Y.T. (Spain) BFM200203068. JCR is a Royal Society University Research Fellow, and would like to thank the Society for all their support. He would also like to thank EDAN for their hospitality, and Iberdrola for their generosity. 


\section{References}

[1] S. Ahmad. On almost periodic solutions of the competing species problem. Proc. Amer. Math. Soc. 102 (1988), 855-861.

[2] S. Ahmad. On the nonautonomous Volterra-Lotka competition equation. Proc. Amer. Math. Soc. 117 (1993), 199-205.

[3] S. Ahmad. Extinction of species in nonautonomous Lotka-Volterra systems. Proc. Amer. Math. Soc. 127 (1999), 2905-2910.

[4] S. Ahmad and A.C. Lazer. On the nonautonomous $N$-competing species problems, App. Anal. 57 (1995), 309-323.

[5] D. N. Cheban, P. E. Kloeden and B. Schmalfuss. The relationship between pullback, forwards and global attractors of non-autonomous dynamical systems. Preprint

[6] V. V. Chepyzhov and M. I. Vishik. Attractors of non-autonomous dynamical systems and their dimension. J. Math. Pures Appl. 73 (1994), 279-333.

[7] I. Chueshov. Order-preserving skew-product flows and non-autonomous parabolic systems. Acta Appl. Math. 65 (2001), 185-205.

[8] W.A. Coppel. Dichotomies in Stability Theory (Berlin: Springer Lecture Notes in Mathematics No. 629, 1978).

[9] H. Crauel. Random point attractors versus random set attractors. J. Lond. Math. Soc. 63 (2001), 413-427.

[10] H. Crauel, A. Debussche and F. Flandoli. Random attractors. J. Dynamics Differential Equations 9 (1997), 397-341.

[11] P. Hess and A.C. Lazer. On an abstract competition model and applications. Nonlinear Anal. TMA 16 (1991), 917-940.

[12] P. E. Kloeden and B. Schmalfuss. Asymptotic behaviour of non-autonomous difference inclusions. Systems \& Control Letters 33 (1998), 275-280.

[13] J.A. Langa, and A. Suárez. Pullback permanence for non-autonomous partial differential equations, Electronic Journal of Differential Equations, 2002 (2002) No. 72, 1-20.

[14] J.A. Langa, J.C. Robinson, and A. Suárez. Stability, instability, and bifurcation phenomena in non-autonomous differential equations, Nonlinearity 15 (2002), 887-903.

[15] J.A. Langa, J.C. Robinson, and A. Suárez. Permanence in the non-autonomous Lotka-Volterra competition model, J. Diff. Eqs., to appear.

[16] N. Malhotra \& S. Wiggins. Geometric structures, lobe dynamics, and Lagrangian transport in flows with aperiodic time-dependence, with applications to Rossby wave flow. J. Nonlinear Sci. 8 (1998), 401-456.

[17] L. Markus. Asymptotically autonomous differential systems. In Contributions to the Theory of Nonlinear Oscillations III (S. Lefschetz, ed.), 17-29. Annals of Mathematics Studies 36, Princeton University Press, 1956.

[18] K. Mischaikow, H. Smith, and H.R. Thieme. Asymptotically autonomous semiflows: chain recurrence and Lyapunov functions. Trans. Amer. Math. Soc. 347 (1995), 1669-1685.

[19] F. Montes de Oca and L. Zeeman. Extinction in nonautonomous competitive Lotka-Volterra systems. Proc. Amer. Math. Soc. 124 (1996), 3677-3687.

[20] J.D. Murray. Mathematical Biology (Springer-Verlag, New York 1993).

[21] R. Redheffer. Nonautonomous Lotka-Volterra systems, I and II. J. Diff. Eqs 127 (1996), 519-541 and 132 (1996), 1-20.

[22] R.J. Sacker and G.R. Sell. Existence of dichotomies and invariant splitting for linear differential systems I, II, III. J. Diff. Eq. 15, 22, 22 (1974, 1976, 1976) 429-458, 478-496, 497-522.

[23] B. Schmalfuss. Attractors for the non-autonomous dynamical systems. Proceedings of Equadiff 99 Berlin (ed. B. Fiedler, K. Gröger and J. Sprekels) 684-689 (Singapore: World Scientific, 2000).

[24] G. Sell. Non-autonomous differential equations and dynamical systems. Trans. Amer. Math. Soc. 127 (1967), 241-283. 
[25] S. Siegmund. Dichotomoy spectrum for nonautonomous differential equations. J. Dyn. Diff. Eq. 14 (2002a), pp. 243-258.

[26] H.L. Smith. Monotone Dynamical Systems (Providence, RI: AMS Math. Surveys and Monographs vol. 41, 1995).

[27] R. Temam. Infinite-Dimensional Dynamical Systems in Mechanics and Physics (New-York: Springer-Verlag, 1988).

[28] H. Thieme. Asymptotically autonomous differential equations in the plane. Rocky Mountain J. Math. 24 (1994), 351-380.

[29] Y. Yi. A generalized integral manifold theorem. J. Diff. Eq. 102 (1993), 153-187. 